\title{
Family ties: Abundances in Terzan 7, a Sgr dSph globular cluster $\star, \star \star$
}

\author{
L. Sbordone ${ }^{1,2}$, P. Bonifacio ${ }^{3}$, G. Marconi ${ }^{1,4}$, R. Buonanno ${ }^{2}$, and S. Zaggia ${ }^{3}$ \\ 1 ESO European Southern Observatory - Alonso de Cordova 3107 Vitacura, Santiago, Chile \\ 2 Universitá di Roma 2 "Tor Vergata" - via della Ricerca Scientifica, Rome, Italy \\ e-mail: sbordone@mporzio.astro.it \\ 3 Istituto Nazionale di Astrofisica - Osservatorio Astronomico di Trieste, via Tiepolo 11, 34131 Trieste, Italy \\ ${ }^{4}$ Istituto Nazionale di Astrofisica - Osservatorio Astronomico di Roma via Frascati 33, 00040 Monte Porzio Catone, Rome, \\ Italy
}

Received 4 November 2004 / Accepted 23 February 2005

\begin{abstract}
We study the chemical composition of 5 giant stars in the globular cluster Terzan 7 associated with the Sagittarius dwarf spheroidal galaxy (Sgr dSph), based on high resolution UVES-VLT spectra. We confirm the metallicity found by previous high resolution investigations: $[\mathrm{Fe} / \mathrm{H}] \sim-0.6$. We also show that this cluster displays the same low $\alpha$-element to iron ratio found in Sgr dSph field stars of similar metallicity, as well as the same low Ni/Fe ratio. These chemical signatures are characteristic of the Sgr dSph system, and appear to be shared both by the globular cluster Pal 12, which was most likely stripped from Sgr by tidal interaction, and by Pal 5, which may also have belonged in the past to the Sgr dSph system. Intriguingly even globular cluster Ruprecht 106, although not associated to Sgr, displays similar characteristics.
\end{abstract}

Key words. stars: abundances - stars: atmospheres - Galaxy: globular clusters: individual: Terzan 7 - galaxies: abundances galaxies: individual: Terzan 7 - galaxies: evolution

\section{Introduction}

Terzan 7 (Terzan 1968; Buonanno et al. 1995) is a sparse, young, and metal rich globular cluster, physically associated to the Sagittarius dwarf spheroidal galaxy (Sgr dSph, Ibata et al. 1995; Marconi et al. 1998). The dwarf galaxy, to which 4 globular clusters are dynamically linked (M 54, Terzan 7, Terzan s8, and Arp 2), is undergoing tidal merging with the Milky Way (MW) Halo, and is gradually being disrupted. Clear detection of the associated stellar stream inside the Halo has been achieved with 2MASS data (Majewski et al. 2003); clear indications exist that some MW globular clusters most likely originated in the Sgr dSph system, and then were stripped and added to the halo GC system (the best candidate being Pal 12, see Cohen 2003).

Terzan 7 has long been known to be a somewhat peculiar globular cluster. Even before the Sgr dSph discovery (Ibata et al. 1995), it has been associated with a small group of other objects due to their anomalous characteristics. It was shown to share with Arp 2, Ruprecht 106 and Pal 12 the young age and a significant discrepancy between the photometric metallicities and those based on the Ca II IR triplet (Buonanno et al. 1995,

\footnotetext{
* Based on observations obtained in ESO programmes 67.B-0147 and 65.L-0481.

$\star \star$ Table 5 is only available in electronic form at http://www. edpsciences.org
}

and references therein). The fact that all these clusters, with the exception of $\mathrm{Ru} 106$, now appear to be somehow linked to the Sgr dSph leads one to interpret their anomalies as linked to their origin in the Sgr dSph system.

We are conducting a comprehensive study of chemical abundances in the Sgr dSph system, in order to clarify its evolutionary characteristics, along with the effect of its interaction with the MW Halo. In previous papers we presented a study of 12 giant stars in the main body of Sgr dSph (Bonifacio et al. 2000; Bonifacio et al. 2004, henceforth Paper I), while in this paper we present a similar analysis of five Terzan 7 giants.

As shown in Paper I, we identified a very young, metal rich population $([\mathrm{Fe} / \mathrm{H}] \sim 0$, age less than $2 \mathrm{GYr})$ in the Sgr $\mathrm{dSph}$. This population was also characterized by relatively low $\alpha$ over iron ratios. Such feature may be interpreted as the signature of a prolonged, slow, or bursting star formation. A trend in $[\alpha / \mathrm{Fe}]$ vs. $[\mathrm{Fe} / \mathrm{H}]$ towards lower $\alpha$ content at high iron content was barely detectable in our sample, but emerged clearly when the abundances of M 54 giants were also included (Brown et al. 1999). This was even more evident by looking at all the available data of the Local Group dwarf galaxies. This trend appears to be significantly different from the one displayed by the MW stars, which leads us to infer a different evolutionary behavior between Local Group dwarf spheroidals and the Milky Way. In this scenario, the chemical composition of Terzan 7 (and of the other globular clusters associated to the Sgr dSph) is of particular importance; since it belongs to the Sgr dSph 
Table 1. Photometry and physical parameters for the five stars.

\begin{tabular}{|c|c|c|c|c|c|c|c|c|}
\hline Number $^{a}$ & $\begin{array}{l}V \\
\mathrm{mag}\end{array}$ & $\begin{array}{l}(B-V)_{0} \\
\operatorname{mag}\end{array}$ & $\begin{array}{l}T_{\text {eff }} \\
\mathrm{K}\end{array}$ & $\begin{array}{l}\log g \\
\operatorname{cgs}\end{array}$ & $\begin{array}{l}\boldsymbol{\xi} \\
\mathrm{km} \mathrm{s}^{-1}\end{array}$ & $\begin{array}{c}\alpha(J 2000) \\
\text { hms }\end{array}$ & $\begin{array}{c}\delta(J 2000) \\
\circ,,, "\end{array}$ & $\begin{array}{c}v_{r}^{b} \\
\mathrm{~km} \mathrm{~s}^{-1}\end{array}$ \\
\hline 1272 & 16.62 & 1.15 & 4421 & 1.2 & 1.45 & 191737.1 & -343911.9 & 158.9 \\
\hline $1282^{c}$ & 16.08 & 1.30 & 4203 & 1.3 & 1.60 & 191739.4 & -343906.4 & 158.2 \\
\hline 1515 & 16.76 & 1.12 & 4468 & 2.0 & 1.45 & 191738.2 & -343916.8 & 157.8 \\
\hline $1665^{d}$ & 15.04 & 1.50 & 3945 & 0.8 & 1.60 & 191743.2 & -343943.2 & 159.6 \\
\hline $1708^{e}$ & 16.08 & 1.28 & 4231 & 1.2 & 1.70 & 191743.5 & -343912.4 & 160.6 \\
\hline
\end{tabular}

${ }^{a}$ Referred to the catalog of Buonanno et al. (1995), available via CDS at http://cdsweb.u-strasbg.fr/cgi-bin/qcat?]/AJ/109/663/

${ }^{b}$ The presented $v_{r}$ is the heliocentric value.

${ }^{c}$ This is star S34 in Tautvaišiené et al. (2004).

${ }^{d}$ This is star S16 in Tautvaišiené et al. (2004).

${ }^{e}$ This is star S35 in Tautvaišiené et al. (2004).

system, we expect to find the same chemical peculiarities we observed in the main body of the galaxy.

Detailed abundances on Terzan 7 stars are presented in the literature only by Tautvaišiené et al. (2004), where the authors analyzed three cool low-gravity stars, one of which could be an AGB due to its very low gravity, and found a rather high mean metallicity $([\mathrm{Fe} / \mathrm{H}] \sim-0.6)$, very slight $\alpha$ enhancement, and a marginally significant $\mathrm{Ni}$ under-abundance with respect to iron. A strong $\mathrm{La}$ and Eu overabundance is also noticeable in their results.

Along with the Sgr dSph main body stars described in Bonifacio et al. (2004), we also observed three giants in Terzan 7 (see Sbordone et al. 2004a). One of the stars of Tautvaišiené et al. (2004) was also observed by us, although with a slightly different instrumental setting. We decided to produce a methodologically coherent analysis of all the five Terzan 7 stars observed at high resolution, looking in particular for the presence of a "family signature" linking Terzan 7 with the Sgr dSph and the other globular clusters associated to the system.

\section{Observations and data reduction}

The five stars studied in this paper have all been observed at the VLT/UVES high resolution spectrograph. Their coordinates, photometry, and atmospheric parameters are listed in Table 1. The first three stars (\# 1272, \# 1515, \# 1282) have been observed in the course of our programme in dichroic 1 (DIC1) mode during the same session devoted to the Sgr dSph main body described in Paper I. Both blue and red arms were used, but only red arm data is presented here. The integration time was $3600 \mathrm{~s}$ on each star, split into two $1800 \mathrm{~s}$ exposures. The pipeline extracted, radial velocity corrected spectra were coadded to reach a $S / N \simeq 50$ at $600 \mathrm{~nm}$, increasing at longer wavelengths.

The spectra for the three stars described in Tautvaišiené et al. (2004) were retrieved from the ESO archive. They are dichroic 2 spectra (DIC2), with both blue and red arm data (blue chip 375 to $500 \mathrm{~nm}$, red CCD mosaic 593 to $960 \mathrm{~nm}$ ). Each star received a single 3600 s exposure. We have extracted the spectra by means of the UVES pipeline, obtaining an $S / N$ of about 70 at $600 \mathrm{~nm}$. Blue DIC 2 chip was not in fact

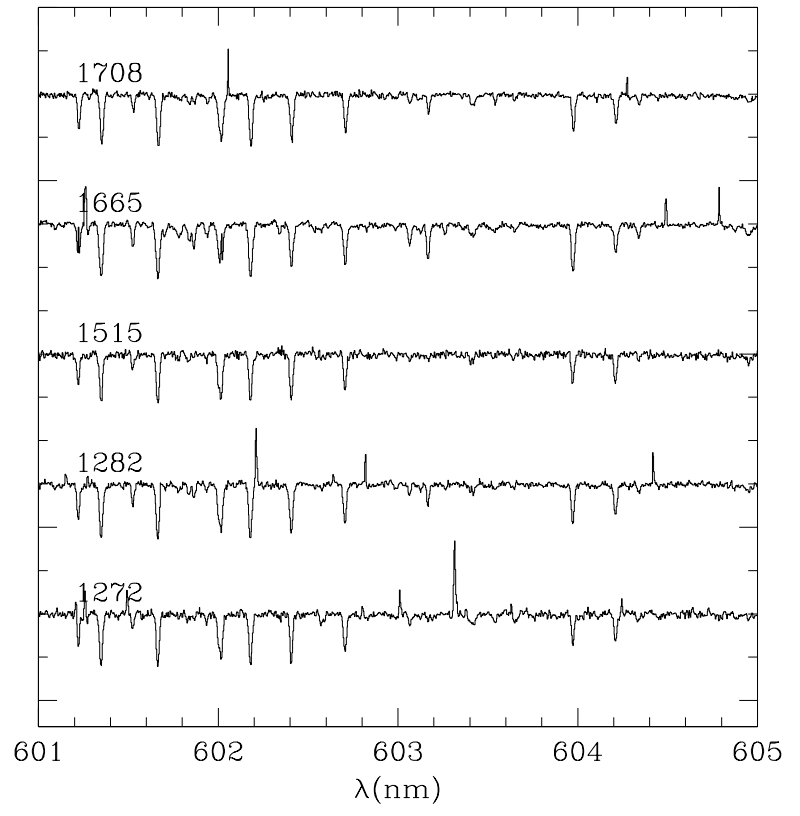

Fig. 1. A sample of the spectra of the five stars in the wavelength range 601 to $605 \mathrm{~nm}$, where many of the considered Fe I features lie. For Star \# 1282 the DIC1 spectrum is shown.

used: some lines in that wavelength range were initially included in the linelist, but in metal rich, low gravity stars that area of the spectrum is subject to such crowding that a proper continuum estimate was deemed very difficult and uncertain.

A sample of the spectra is shown in Fig. 1. The star identified as S34 in Tautvaišiené et al. (2004) is Star \# 1282, also observed independently by us.

For all the stars, equivalent widths $(E W s)$ were measured for lines of MgI, SiI, CaI, TiI, Fe I, Fe II and Ni I by using IRAF task splot; see Table 5 for details. The lines employed are essentially the same used in Paper I for \# 1515 and \# 1272, while a completely different linelist was prepared for stars \# 1665 and \# 1708, due to the different spectral coverage. Star \# 1282, having both DIC1 and DIC2 spectra available, had the two linelists merged. In the (sporadic) case of a line being measured in both spectra, the mean of the two $E W \mathrm{~s}$ was used. 
Table 2. Abundances: $S / N$ are per pixel. On Star \# 1282 the values are DIC1 and DIC2, respectively.

\begin{tabular}{|c|c|c|c|c|c|c|c|c|c|c|c|c|c|c|c|}
\hline Star & $\begin{array}{l}S / N \\
@ 650 \mathrm{~nm}\end{array}$ & $A(\mathrm{Fe} \mathrm{I})$ & $n$ & $A(\mathrm{Fe}$ II $)$ & $n$ & $A(\mathrm{Mg})$ & $n$ & $A(\mathrm{Si})$ & $n$ & $A(\mathrm{Ca})$ & $n$ & $A(\mathrm{Ti})$ & $n$ & $A(\mathrm{Ni})$ & $n$ \\
\hline Sun & & 7.50 & & 7.50 & & 7.58 & & 7.55 & & 6.36 & & 6.36 & & 6.36 & \\
\hline 1272 & 43 & $6.83 \pm 0.08$ & 12 & $6.85 \pm 0.06$ & 4 & $6.88 \pm 0.13$ & 4 & $6.91 \pm 0.16$ & 4 & $5.88 \pm 0.09$ & 8 & $4.47 \pm 0.09$ & 5 & $5.32 \pm 0.09$ & 5 \\
\hline 1282 & $55 / 65$ & $6.96 \pm 0.11$ & 41 & $6.92 \pm 0.10$ & 12 & $6.88 \pm 0.05$ & 5 & $7.06 \pm 0.11$ & 8 & $5.74 \pm 0.09$ & 8 & $4.50 \pm 0.13$ & 16 & $5.47 \pm 0.08$ & 12 \\
\hline 1515 & 45 & $6.94 \pm 0.09$ & 11 & $6.89 \pm 0.08$ & 4 & $6.99 \pm 0.09$ & 3 & $7.14 \pm 0.05$ & 5 & $5.85 \pm 0.10$ & 9 & $4.52 \pm 0.07$ & 6 & $5.54 \pm 0.05$ & 6 \\
\hline 1665 & 60 & $6.99 \pm 0.14$ & 33 & $6.98 \pm 0.09$ & 8 & $6.93 \pm 0.12$ & 3 & $7.00 \pm 0.12$ & 8 & $5.79 \pm 0.11$ & 5 & $4.59 \pm 0.21$ & 11 & $5.49 \pm 0.13$ & 8 \\
\hline 1708 & 65 & $6.94 \pm 0.12$ & 34 & $6.96 \pm 0.06$ & 5 & $6.84 \pm 0.08$ & 3 & $6.98 \pm 0.20$ & 6 & $5.65 \pm 0.05$ & 5 & $4.39 \pm 0.11$ & 16 & $5.52 \pm 0.11$ & 15 \\
\hline
\end{tabular}

\section{Abundance analysis}

The analysis method is the one already described in Paper I: the effective temperatures have been derived from $(B-V)_{0}$ colors using the calibration by Alonso et al. (1999); we assumed $E(B-V)=0.07$ as in Marconi et al. (1998). Notice that the calibration provided in Alonso et al. (1999) for $(B-V)_{0}$ colors is marginally dependent on the star's $[\mathrm{Fe} / \mathrm{H}]$. For verification, we first derived $T_{\text {eff }}$ assuming $[\mathrm{Fe} / \mathrm{H}]=-0.6$, and then recalculated by using the derived metallicity. The variations were totally negligible, on the level of a few $\mathrm{K}$.

Tautvaišiené et al. (2004) used a different approach to $T_{\text {eff }}$ determination and relied on an excitation temperature; nevertheless their estimates result in good agreement with our $T_{\text {eff }}$ 's, differing at most $50 \mathrm{~K}$. Estimates of the effect of systematics in the physical parameters are given in Table 3.

Gravity was initially estimated from a comparison with Padova isochrones (Salasnich et al. 2000). One-dimensional, LTE models were calculated by using the ATLAS 9 code (Kurucz 1993). The abundances were extracted from the measured $E W$ s by using WIDTH; spectral syntheses were produced with SYNTHE (for both codes see Kurucz 1993). SYNTHE was first used in the DIC 2 linelist selection phase when it helped in selecting unblended lines of adequate strength, and then after the analysis, as a check of internal consistency by verifying correspondence between the measured lines and their syntheses. For all these codes, our Linux ported versions were used, as described in Sbordone et al. (2004c).

The models were calculated without introducing overshooting (see Castelli et al. 1997), and with solar scaled abundances, since no significant $\alpha$-enhancement was found. Where necessary, the original estimated gravity has been adjusted in order to satisfy the iron ionization equilibrium. The changes were generally small, on the order of $\Delta \log g \sim 0.1$. A notable exception is Star \# 1272 which, with magnitude and colours similar to Star \# 1515, has a gravity coherent with stars that are almost 0.5 mag brighter in $V$ and 0.15 mag redder in $(B-V)$, like, for example, Star \# 1282. For Star \# 1272 the spectroscopic gravity is therefore about 0.8 dex lower than the one suggested by the isochrones.

The microturbulence was determined, as usual, by requiring no correlation between the $E W$ and the derived abundance for each Fe I line.

For one star, \# 1665, significant inconsistencies emerged between the abundances computed with WIDTH and the corresponding synthetic spectra computed with SYNTHE. Examination of the results showed that this was due to the fact that SYNTHE read as input a model in which the elemental abundances had been changed according to the results of WIDTH, whereas WIDTH read a copy of the model in which all abundances were solar scaled. This is common practice in abundance analysis using ODF-based model atmospheres: one computes a model-atmosphere with a given ODF and the corresponding set of abundances, then with this model atmosphere one computes the detailed line transfer (with SYNTHE, WIDTH or similar codes), varying the elemental abundances until a match is found between computed and observed spec$\operatorname{tra} / E W \mathrm{~s}$.

This practice is legitimate inasmuch as the abundance variation from the set assumed in the model computation has little or no influence on the model structure. While true for trace elements, it is in general not true for $\alpha$ elements, as pointed out by Bonifacio \& Caffau (2003). Indeed, both the WIDTH and the SYNTHE suite, upon reading the input model atmosphere and abundances, compute the number densities of atomic species at all depths. Thus while a change in Fe abundance will have a negligible effect on the model structure, a change in $\mathrm{Mg}$ abundance will result in a change in the model ionization structure, since $\mathrm{Mg}$ is the main electron donor. It must be stressed that in such cases the results obtained by computing synthetic spectra from a model computed with a different chemical composition are inconsistent. On the other hand, in those cases in which e.g. a computation of abundance with WIDTH, yields the same result both when solar scaled abundances are used as input (and thus in the computation of number densities) and when the abundances so derived are used as input, it is justified to assume that the abundance of the given element has no significant effect on the model structure.

This assumption is indeed adequate for all the stars in our sample except Star \# 1665. For this star we computed an ad-hoc opacity sampling model with observed abundances by using ATLAS 12 (Kurucz 1993). In Fig. 3 we show the temperature and ionization structure for models appropriate for Star \# 1665 in three cases: 1) ATLAS 12 opacity sampling model with abundances given in Table 2; 2) ATLAS 9 ODF-based model with solar scaled abundances; 3) ATLAS 9 ODF-based model with abundances given in Table 2 . It is obvious that changing the abundances in the ATLAS 9 model alters the ionization structure, which is, however, different from the one consistently computed with ATLAS 12 when all the final abundances are taken into account during the model computation. From Fig. 3 one may appreciate that using the different models would imply a difference in derived $\mathrm{Mg}$ abundance on the order of 0.2 dex. The process is clearly iterative, as one starts from abundances derived from a model with solar-scaled abundances, then uses 
Table 3. Errors in the abundances of Star \# 1282 due to uncertainties in the atmospheric parameters.

\begin{tabular}{|c|c|c|c|c|c|c|c|}
\hline & $\Delta A(\mathrm{Fe} \mathrm{I})$ & $\Delta A(\mathrm{Fe} \mathrm{II})$ & $\Delta A(\mathrm{Mg})$ & $\Delta A(\mathrm{Si})$ & $\Delta A(\mathrm{Ca})$ & $\Delta A(\mathrm{Ti})$ & $\Delta A(\mathrm{Ni})$ \\
\hline$\Delta \xi= \pm 0.2 \mathrm{~km} \mathrm{~s}^{-1}$ & $\begin{array}{l}-0.07 \\
+0.08\end{array}$ & $\begin{array}{l}-0.05 \\
+0.04\end{array}$ & $\begin{array}{l}-0.03 \\
-0.04\end{array}$ & $\mp 0.03$ & $\begin{array}{l}-0.12 \\
+0.13\end{array}$ & $\begin{array}{l}-0.09 \\
+0.10\end{array}$ & $\mp 0.07$ \\
\hline$\Delta T_{\mathrm{eff}}= \pm 100 \mathrm{~K}$ & $\begin{array}{l}+0.01 \\
+0.00\end{array}$ & $\begin{array}{l}-0.15 \\
+0.16\end{array}$ & $\begin{array}{l}+0.03 \\
+0.00\end{array}$ & $\begin{array}{l}-0.08 \\
+0.11\end{array}$ & $\begin{array}{l}+0.11 \\
-0.12\end{array}$ & $\begin{array}{l}+0.18 \\
-0.17\end{array}$ & $\begin{array}{l}+0.00 \\
-0.01\end{array}$ \\
\hline$\Delta T_{\text {eff }}= \pm 100 K \Delta \log g= \pm 0.20$ & $\begin{array}{l}+0.07 \\
-0.04\end{array}$ & $\begin{array}{l}+0.03 \\
+0.04\end{array}$ & $\begin{array}{l}+0.04 \\
-0.02\end{array}$ & $\begin{array}{l}+0.00 \\
+0.04\end{array}$ & \pm 0.11 & \pm 0.019 & $\begin{array}{l}+0.08 \\
-0.05\end{array}$ \\
\hline$\Delta \log g= \pm 0.50$ & $\begin{array}{l}+0.09 \\
{ }_{-0.10}\end{array}$ & $\begin{array}{l}+0.24 \\
{ }_{-0.30}\end{array}$ & $\begin{array}{l}+0.04 \\
-0.03\end{array}$ & $\begin{array}{l}+0.12 \\
-0.14 \\
\end{array}$ & $\begin{array}{l}-0.01 \\
+0.00 \\
\end{array}$ & $\begin{array}{l}+0.02 \\
-0.03\end{array}$ & $\begin{array}{l}+0.11 \\
-0.13 \\
\end{array}$ \\
\hline
\end{tabular}

Table 4. Abundance ratios. The mean cluster values are obtained by weighted mean.

\begin{tabular}{lcrrrrr}
\hline \hline Star & {$[\mathrm{Fe} / \mathrm{H}]$} & {$[\mathrm{Mg} / \mathrm{Fe}]$} & {$[\mathrm{Si} / \mathrm{Fe}]$} & {$[\mathrm{Ca} / \mathrm{Fe}]$} & {$[\mathrm{Ti} / \mathrm{Fe}]$} & {$[\mathrm{Ni} / \mathrm{Fe}]$} \\
\hline 1272 & $-0.67 \pm 0.08$ & $-0.03 \pm 0.15$ & $0.03 \pm 0.18$ & $0.19 \pm 0.12$ & $0.12 \pm 0.12$ & $-0.26 \pm 0.12$ \\
1282 & $-0.54 \pm 0.11$ & $-0.16 \pm 0.12$ & $0.05 \pm 0.16$ & $-0.08 \pm 0.14$ & $0.02 \pm 0.17$ & $-0.24 \pm 0.14$ \\
1515 & $-0.56 \pm 0.09$ & $-0.03 \pm 0.13$ & $0.15 \pm 0.10$ & $0.05 \pm 0.13$ & $0.06 \pm 0.11$ & $-0.15 \pm 0.10$ \\
1665 & $-0.51 \pm 0.14$ & $-0.14 \pm 0.18$ & $-0.04 \pm 0.18$ & $-0.06 \pm 0.18$ & $0.08 \pm 0.25$ & $-0.25 \pm 0.19$ \\
1708 & $-0.56 \pm 0.12$ & $-0.18 \pm 0.14$ & $-0.01 \pm 0.23$ & $-0.15 \pm 0.13$ & $-0.07 \pm 0.16$ & $-0.17 \pm 0.16$ \\
Mean & $\mathbf{- 0 . 5 9} \pm \mathbf{0 . 0 7}$ & $\mathbf{- 0 . 1 1} \pm \mathbf{0 . 0 7}$ & $\mathbf{0 . 0 7} \pm \mathbf{0 . 0 9}$ & $\mathbf{- 0 . 0 0} \pm \mathbf{0 . 1 4}$ & $\mathbf{- 0 . 0 5} \pm \mathbf{0 . 0 7}$ & $\mathbf{- 0 . 1 9} \pm \mathbf{0 . 0 5}$ \\
\hline
\end{tabular}

these to compute an ATLAS 12 model and finally determines new abundances, to be used for a new ATLAS 12 model; and so on, until the derived abundances coincide with the abundances used in the model computation. In our case two iterations were sufficient. We want to stress that the need for computing an ATLAS 12 model for this star is due to its extremely low gravity; nevertheless, the derived abundances appear in reassuring concordance with the ones derived for the other stars.

The atomic data used in the analysis are provided in Table 5; they are almost always the same as in Paper I (derived from the Castelli - Kurucz database, Castelli \& Kurucz 2003) except for $\mathrm{Mg}$ I, for which we adopted the $\log g f$ values derived by Gratton et al. (2003) from measured lifetimes and theoretical branching ratios, and $\mathrm{Ca}$ I, for which we adopted the furnace measurements of Smith \& Raggett (1981). All the lines have either experimental or theoretical $\log (g f)$ values, with the exception of four Si I lines (marked as ED in Table 5) which have oscillator strengths derived from solar spectra (Edvardsson et al. 1993).

\section{Results}

The derived abundances are listed in Table 2 and the corresponding abundance ratios in Table 4 . The associated errors are the scattering between the abundance given by the single lines and their propagation in the abundance ratios. Iron abundances appear to be in very good agreement with the results in Tautvaišiené et al. (2004), leading to a mean value of $[\mathrm{Fe} / \mathrm{H}] \sim-0.6$. Our analysis provides $[\mathrm{Mg} / \mathrm{Fe}]=-0.12$ for star \# 1665 and -0.10 for \# 1708, coherent with the values we found for the first three stars. However this is significantly at odds with the Tautvaišiené et al. (2004) results. Leaving aside the case of Star \# 1665 due to its very low gravity and temperature, mean $\mathrm{Mg}$ and $\mathrm{Ti}$ abundances in Tautvaišiené et al. (2004) are about 0.25 dex higher than ours, while their Si abundance is 0.17 dex lower than the one we obtained.

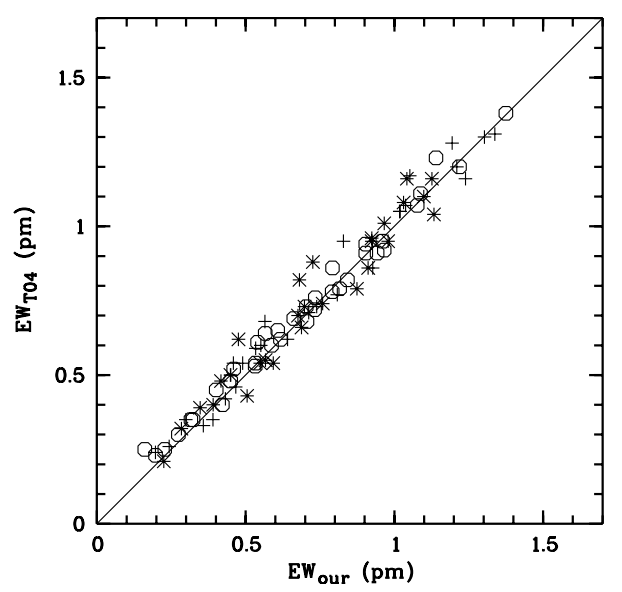

Fig. 2. Comparison of the equivalent widths measured by us and by Tautvaišiené et al. (2004) for the lines in common. Open hexagons refer to Star \# 1282, crosses refer to Star \# 1665 and asterisks to Star \# 1708. The straight line is not a fit, but simply the bisector.

The three stars in common testify that no significant offset is introduced by the different $T_{\text {eff }}$ calibration methods. The (almost) identical gravities derived starting with (almost) identical iron abundances lead us to infer that little difference should exist between the structure of our ATLAS model and of the MARCS ones used in Tautvaišiené et al. (2004). A comparison of $E W \mathrm{~s}$ for lines in common suggests that there is no significant offset in $E W$ measurements either: a linear regression provides slope of about 1 and no significant offset with an rms, which is on the order of our measurement error (see Fig. 2). The lines in common between the two samples were 1 for $\mathrm{MgI}, 3$ for Si I, 4 for Ti I, 2 for Ca I, 7 for Fe I, 8 for Fe II, and 11 for Ni I. Therefore probably the cause of this discrepancy resides in the choice of lines used and the atomic data. 


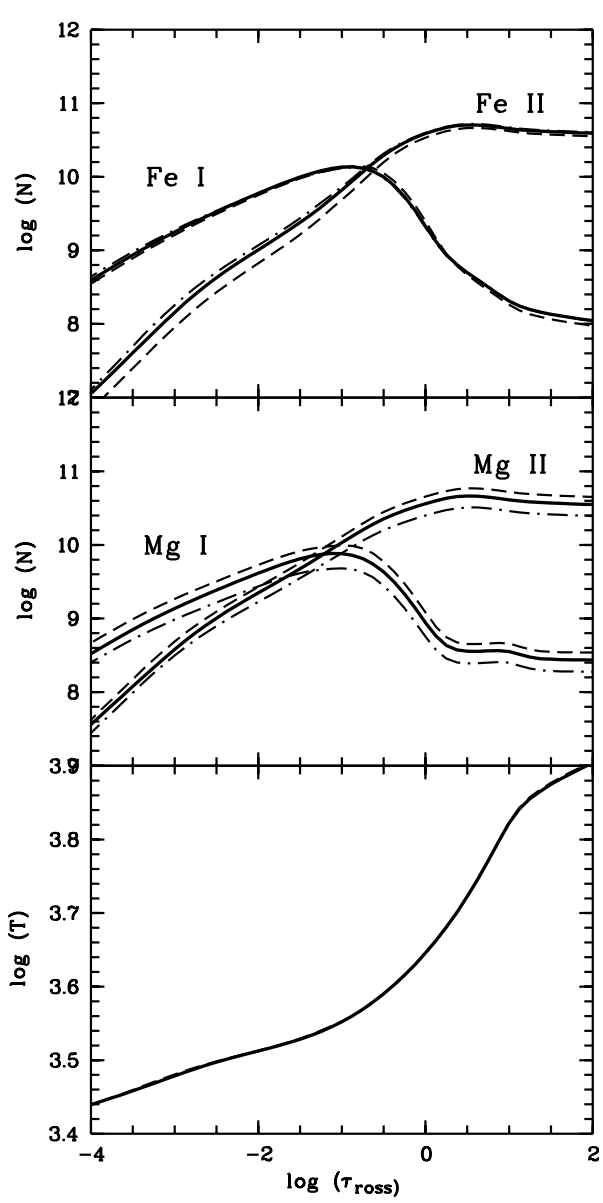

Fig. 3. Atmospheric models for Star \# 1665. All models correspond to $T_{\text {eff }}=3945 \mathrm{~K} \log g=0.80$. The solid lines refer to an opacity sampling ATLAS 12 model with abundances as provided in Table 2. The dashed lines refer to an ATLAS 9 model computed with a solar-scaled ODF with $[\mathrm{M} / \mathrm{H}]=-0.5$ and a microturbulence of $1 \mathrm{~km} \mathrm{~s}^{-1}$. The dash-dotted lines refer to the ionization structure of the ATLAS 9 model computed by WIDTH when the abundances of Table 2 are provided as input. The top panel shows the Fe I and Fe II ions, the middle panel the Mg I and Mg II ions, while the bottom panel displays the temperature structure of the model. The neutral ions show a peak in number densities around $\log \tau_{\text {ross }} \sim-1.5$.

\section{Implications for Terzan 7 origin and evolution}

\section{1. $\alpha$ elements: $\mathrm{Mg}, \mathrm{Si}, \mathrm{Ca}, \mathrm{Ti}$}

These elements show no enhancement with respect to iron. From Fig. 4 one may also see that there is a hint of dispersion in the abundances of $\alpha$ elements within the cluster. Such a dispersion would be unusual in such a small globular cluster, so that given the size of the errors and the small number of analyzed stars, for the time being we are reluctant to claim it as real. A larger sample would be necessary to clarify the situation.

The tendency of dwarf galaxies to show low alpha content (at any given metallicity) compared to the MW is recently emerging as a distinctive feature of their chemical composition (Tolstoy et al. 2003; Venn et al. 2004). As shown in Fig. 4, Terzan 7 stars appear to follow perfectly the same $[\alpha / \mathrm{Fe}]$ vs. $[\mathrm{Fe} / \mathrm{H}]$ path as the Sgr dSph body and M 54, which is completely included in the nucleus of the Sgr dSph: see

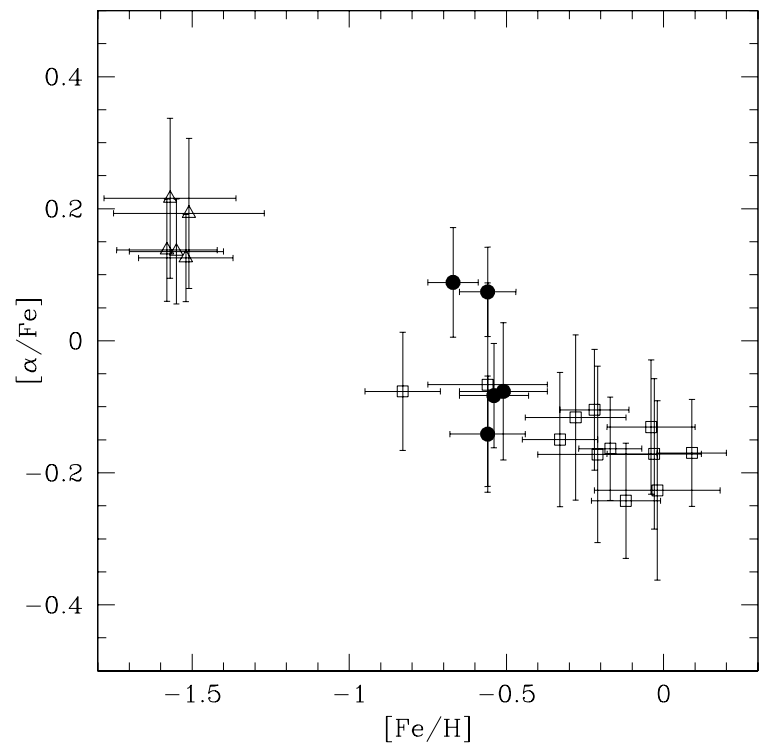

Fig. 4. $[\alpha / \mathrm{Fe}]$ vs. $[\mathrm{Fe} / \mathrm{H}]$ for the five stars of Terzan 7 (filled dots), the 12 stars in the Sgr dSph main body studied in Paper I (open squares), and the five stars in M 54 from Brown et al. (1999) (open triangles). $\alpha$ is defined as the mean of $\mathrm{Mg}, \mathrm{Si}$, and $\mathrm{Ca}$.

Monaco et al. (2003). As shown in Paper I, the same pattern is also followed by the other LG dwarf galaxies, which is strongly hints at common origins for Terzan 7 and the Sgr dSph main body in an environment with low star formation rate along a prolonged time. It is also worth noticing that strongly similar results have been reported for Pal 12 (Cohen 2003), which is supposed to have formed inside the Sgr dSph and consequently been stripped by the MW (Bellazzini et al. 2003). Indeed, the strong chemical similarities between Pal 12 and the Sgr dSph are considered by Cohen et al. as strongly enforcing this theory of the origin of the cluster.

Similar chemical characteristics are also shared by Ruprecht 106 (Brown et al. 1997) and Pal 5 (Smith et al. 2002). These clusters do not have orbital characteristics compatible with a recent stripping from the Sgr dSph, although Pal 5 orbit may be compatible with an older stripping event; see for this cluster Odenkirchen et al. (2003). Nevertheless, they are generally considered to have been accreted by MW from some merging event.

\subsection{Nickel}

As an "iron peak" element, $\mathrm{Ni}$ is usually considered as enriching the interstellar medium through the same processes as iron does and, as a consequence, showing a stable ratio with Fe. This is typically true in Milky Way field stars and in the large majority of galactic globular clusters (Cayrel et al. 2003; Sneden 2004, and references therein). Some significant exceptions exist and have been known for some time, the most notable being again Pal 12 (Cohen 2003) and Ru 106 (Sneden 2004; Brown et al. 1997).

Measurements are now also known for Terzan 7 (this paper and Tautvaišiené et al. 2004) and the Sgr dSph body 
(Sbordone et al. 2004b; Sbordone et al. 2004d; and Bonifacio et al. 2000). In all these objects $\mathrm{Ni}$ appears to be systematically underabundant with respect to iron of amounts between 0.1 to 0.3 dex. Even if slight, this underabundance appears to significantly exceed the spread encountered inside the Milky Way. We have no hypothesis for the possible cause for this underabundance, but, again, we find here a very distinctive signature that ties Ter 7 (and Pal 12, of course) to the Sgr dSph.

\section{Discussion}

We believe that convincing evidence exists that the chemical composition of the Sgr dSph correlates strongly with that of its present or past (supposed) satellites. For the objects presently not dynamically linked to the dwarf galaxy (Pal 12 and maybe Pal 5) this chemical affinity can also be considered as strongly supporting the actual existence of a past connection. Intriguingly Ru 106 is not supposed to be associated to the Sgr dSph, nevertheless it shows a very similar composition pattern. Although an "extreme" object among the LG dwarf galaxies due to its high metallicity, the Sgr dSph seems to share with them a common evolutionary path. If Ru 106 were the residual of another merging process, this similarity would enforce the interpretation that the chemical composition of Sgr dSph is actually not peculiar at all. The problem remains to explain how this abundance pattern actually arises. The Sgr dSph system interacts strongly with the MW, and its mass, likely, decreased significantly in the process. Its ability to retain the SN yields (and more generally the star-forming gas) is consequently difficult to state without detailed simulations. The timescale of their release (faster for type II SNe, Tinsley 1979; Smecker-Hane \& Wyse 1992; Kobayashi et al. 1998) may also have played a role. A final interesting aspect is the formation time of the various globular clusters. M 54, Pal 5, Pal 12, and Ter 7 lie (more or less) equally spaced along the $[\alpha / \mathrm{Fe}]$ vs. $[\mathrm{Fe} / \mathrm{H}]$ graph, leading one to think that they should have formed one after the other throughout a considerable time span, as long as $[\mathrm{Fe} / \mathrm{H}]$ may be considered to be a proxy of time. How could a small object like Sgr dSph continue to form globular clusters while evolving along almost $1 \mathrm{dex}$ in $[\mathrm{Fe} / \mathrm{H}]$ ? The strong interaction with the MW may be an explanation, that might be telling us something about the mechanisms of GC formation during interactions.

Acknowledgements. We are grateful to Fiorella Castelli for many interesting discussions on model-atmospheres and for guiding us in the use of ATLAS12. This research was done with support from the Italian MIUR COFIN2002 grant "Stellar populations in the Local Group as a tool to understand galaxy formation and evolution" (P.I. M. Tosi).

\section{References}

Alonso, A., Arribas, S., \& Martinez-Rogers, C. 1999, A\&AS, 140, 261

Bellazzini, M., Ferraro, F. R., \& Ibata, R. A. 2003, AJ, 125, 188B

Bonifacio, P., \& Caffau, E. 2003, A\&A, 399, 1183

Bonifacio, P., Hill, V., Molaro, P., et al. 2000, A\&A, 359, 663

Bonifacio, P., Sbordone, L., Marconi, G., Pasquini, L., \& Hill, V. 2004, A\&A, 414, 503
Brown, J. A., Wallerstein, G., \& Zucker, D. 1997, AJ, 114, 180

Brown, J. A., Wallerstein, G., \& Gonzalez, G. 1999, AJ, 118, 1245

Buonanno, R., Corsi, C. E., Pulone, L., et al. 1995, AJ, 109, 663

Cayrel, et al. 2003, A\&A, in press

Castelli, F., \& Kurucz, R. L. 2003, Modelling of Stellar Asmospheres, ed. N. Piskunov, et al., Proc. IAU Symp., 210, poster A20 on the enclosed CD-ROM [arXiv: astro-ph/0405087]

Castelli, F., Gratton, R. G., \& Kurucz, R. L. 1997, A\&A, 318, 841

Cohen, J. 2003 [arXiv: astro-ph/0311187]

Edvardsson, B., Andersen, J., Gustafsson, B., et al. 1993, A\&AS, 102, 603

Fuhr, J. R., Martin, G. A., \& Wiese, W. L. 1988, J. Phys. Chem. Ref. Data, 17, Suppl. 4

Garz, T. 1973, A\&A, 26, 471

Gratton, R. G., Carretta, E., Claudi, R., Lucatello, S., \& Barbieri, M. 2003, A\&A, 404, 187

Hannaford, P., Lowe, R. M., Grevesse, N., \& Noels, A. 1992 A\&A, 259,301

Ibata, R. A., Gilmore, G., \& Irwin, M. J. 1995, MNRAS, 277, 781I

Kobayashi, C., Tsujimoto, T., Nomoto, K., Hachisu, I., \& Kato, M. 1998, ApJ, 503, L155

Kurucz, R. L. 1993, CDROM, 13, 18

Kurucz, R. L. 1988, Trans. IAU, XXB, M. McNally, ed., Dordrecht: Kluwer, 168

Kurucz, R. L., \& Peytremann, E. 1975, SAO Special Report 362

Majewski, S. R., Skrutskie, M. F., Weinberg, M. D., \& Ostheimer, J. C. 2003, ApJ, 599, 1082

Marconi, G., Buananno, R., Castellani, M., et al. 1998, A\&A, 330, 453

Martin, G. A., Fuhr, J. R., \& Wiese, W. L. 1988, J. Phys. Chem. Ref. Data 17, Suppl. 3

Monaco, L., Bellazzini, M., Ferraro, F., \& Pancino, E. 2003, in Satellites and Tidal Streams, Proc. Conf. held in La Palma 26-30 May 2003, Spain, ed. F. Prada, D. Martinez-Delgado, \& T. Mahoney [arXiv:astro-ph/0309044]

Odenkirchen, M., Grebel, E. K., Dehnen, W., et al. 2003, AJ, 126, 2385

Salasnich, B., Girardi, L., Weiss, A., \& Chiosi, C. 2000, A\&A, 361, 1023

Sbordone, L., Bonifacio, P., Marconi, G., \& Buonanno, R. 2004a, Mem. SAIt, 75, 396

Sbordone, L., Bonifacio, P., Marconi, G., \& Pasquini, L. 2004b, in preparation

Sbordone, L., Bonifacio, P., Castelli, F., \& Kurucz, R. L. 2004c, MSAIS, 5, 93

Sbordone, L., et al. 2004d, Proc. ESO-Arcetri Conf., Chemical enrichment and mixing in stars in the Milky Way and its satellites, ed. L. Pasquini, \& S. Randich (Berlin: Springer-Verlag), in press

Schulz-Gulde, E. 1969, JQSRT 9, 13

Smecker-Hane, T. A., \& Wyse, R. F. G. 1992, AJ, 103, 1621

Smith, G., \& Raggett, D. S. J. 1981, J. Phys. B Atomic Molecular Phys., 14, 4015

Smith, G. H., Sneden, C., \& Kraft, R. P. 2002, AJ, 123, 1502

Sneden, C. 2004, Mem. SAIt, 75, 267

Tautvaišiené, G., Wallerstein, G., Geisler, D., Gonzales, G., \& Charbonnel, C. 2004, AJ, 127, 373

Terzan, A. 1968, C.R. Acad. Sci. Ser. B1, 267, 1245

Tinsley, B. M. 1979, ApJ, 229, 1046

Tolstoy, E., Venn, K., Shetrone, M., et al. 2003, AJ, 125, 707

Venn, K. A., Irwin, M., Shetrone, M. D., et al. 2004, AJ, 128, 1177

Wickliffe, M. E., \& Lawler, J. E. 1997, ApJS, 110, 163

Wiese, W. L., Smith, M. W., \& Glennon, B. M. 1966, NSRDS-NBS 4

Wallerstein, G., Gonzalez, G., \& Geisler, D. 2002, IAUSS, 207, 171 


\section{Online Material}


Table 5. Lines.

\begin{tabular}{|c|c|c|c|c|c|c|c|c|c|c|c|c|c|}
\hline Ion & $\begin{array}{r}\lambda \\
(\mathrm{nm})\end{array}$ & $\log g f$ & $\begin{array}{l}\text { Source of } \\
\log g f \\
\text { (see notes) }\end{array}$ & $\begin{array}{r}E W \\
(\mathrm{pm})^{a} \\
1272\end{array}$ & $\epsilon$ & $\begin{array}{r}E W \\
(\mathrm{pm})^{a} \\
1282\end{array}$ & $\epsilon$ & $\begin{array}{r}E W \\
(\mathrm{pm})^{a} \\
1515\end{array}$ & $\bar{\epsilon}$ & $\begin{array}{r}E W \\
(\mathrm{pm})^{a} \\
1665\end{array}$ & $\epsilon$ & $\begin{array}{r}E W \\
(\mathrm{pm})^{a} \\
1708\end{array}$ & $\bar{\epsilon}$ \\
\hline $\mathrm{Fe} I$ & 466.1535 & -1.270 & FMW & - & - & 4.21 & 6.68 & - & - & 6.53 & 7.07 & - & - \\
\hline $\mathrm{Fe} I$ & 479.4354 & -4.050 & FMW & - & - & 5.90 & 6.94 & - & - & 6.25 & 6.77 & - & - \\
\hline $\mathrm{Fe}_{\mathrm{I}}$ & 484.9668 & -2.680 & FMW & - & - & - & - & - & - & 4.50 & 6.70 & - & - \\
\hline $\mathrm{Fe}_{\mathrm{I}}$ & 489.2859 & -1.290 & FMW & 7.11 & 6.923 & 7.15 & 6.82 & 6.51 & 6.90 & - & - & - & - \\
\hline $\mathrm{Fe} I$ & 489.6439 & -2.050 & FMW & - & - & 7.20 & 7.15 & - & - & 7.80 & 7.16 & - & - \\
\hline $\mathrm{Fe} I$ & 491.8013 & -1.360 & FMW & - & - & 6.41 & 6.75 & - & - & 6.77 & 6.73 & - & - \\
\hline $\mathrm{Fe} I$ & 510.9652 & -0.980 & FMW & 8.24 & 6.94 & - & - & 8.24 & 7.03 & - & - & - & - \\
\hline $\mathrm{Fe} I$ & 552.5544 & -1.330 & FMW & 7.32 & 6.95 & 8.05 & 6.99 & 6.66 & 6.93 & - & _- & _- & - \\
\hline $\mathrm{Fe} I$ & 585.6087 & -1.640 & FMW & 4.11 & 6.71 & 5.84 & 6.96 & 4.20 & 6.84 & - & - & - & - \\
\hline $\mathrm{Fe} I$ & 585.8779 & -2.260 & FMW & 2.26 & 6.84 & 3.55 & 7.05 & 1.95 & 6.86 & - & - & - & - \\
\hline $\mathrm{Fe} I$ & 586.1109 & -2.450 & FMW & 1.44 & 6.87 & - & - & - & - & - & - & - & - \\
\hline $\mathrm{Fe} I$ & 587.7794 & -2.230 & FMW & - & - & - & - & 3.24 & 7.09 & - & - & - & - \\
\hline $\mathrm{Fe} I$ & 588.3817 & -1.360 & FMW & 7.83 & 6.71 & 9.72 & 6.98 & 7.89 & 6.85 & - & - & - & - \\
\hline Fe I & 595.2717 & -1.440 & FMW & - & - & 9.04 & 6.95 & - & - & 8.37 & 6.70 & 9.59 & 6.98 \\
\hline $\mathrm{Fe} I$ & 601.2210 & -4.200 & FMW & - & - & 8.38 & 7.14 & - & - & - & - & 7.83 & 6.99 \\
\hline $\mathrm{Fe} I$ & 601.5245 & -4.680 & FMW & - & - & - & - & - & - & - & - & 3.44 & 6.76 \\
\hline $\mathrm{Fe} I$ & 601.9366 & -3.360 & FMW & - & - & - & - & - & - & 3.09 & 7.05 & 1.93 & 6.92 \\
\hline $\mathrm{Fe}_{\mathrm{I}}$ & 602.4058 & -0.120 & FMW & - & - & 11.97 & 6.95 & - & - & 11.65 & 6.82 & 11.97 & 6.86 \\
\hline $\mathrm{Fe}_{\mathrm{I}}$ & 602.7051 & -1.210 & FMW & - & - & 9.66 & 6.96 & - & - & 10.18 & 6.95 & 9.66 & 6.87 \\
\hline $\mathrm{Fe} I$ & 605.6005 & -0.460 & FMW & - & - & 7.92 & 6.74 & - & - & 8.29 & 6.76 & 9.24 & 6.92 \\
\hline $\mathrm{Fe} I$ & 607.9008 & -1.120 & FMW & - & - & 6.07 & 6.94 & - & _- & - & - & 7.02 & 7.06 \\
\hline $\mathrm{Fe} I$ & 609.6663 & -1.930 & FMW & - & - & 6.65 & 6.96 & - & - & 7.28 & 6.97 & 6.79 & 6.94 \\
\hline $\mathrm{Fe}_{\mathrm{I}}$ & 610.5128 & -2.050 & FMW & - & - & 2.28 & 6.99 & - & - & 3.19 & 7.11 & 2.38 & 6.99 \\
\hline $\mathrm{Fe} I$ & 612.0246 & -5.950 & FMW & - & - & - & - & - & - & - & - & 6.37 & 6.67 \\
\hline $\mathrm{Fe} I$ & 615.1616 & -3.299 & FMW & 10.45 & 6.75 & - & - & 9.99 & 6.85 & - & - & - & - \\
\hline $\mathrm{Fe} I$ & 615.9375 & -1.970 & FMW & - & - & - & - & - & - & - & - & 2.93 & 7.11 \\
\hline $\mathrm{Fe} I$ & 616.5360 & -1.550 & FMW & 6.11 & 6.78 & 7.06 & 6.87 & 6.24 & 6.92 & 8.08 & 6.96 & 7.26 & 6.85 \\
\hline $\mathrm{Fe}_{\mathrm{I}}$ & 618.7989 & -1.720 & FMW & 6.77 & 6.81 & 8.20 & 6.98 & - & - & 8.64 & 6.95 & 7.72 & 6.83 \\
\hline $\mathrm{Fe} I$ & 622.6734 & -2.220 & FMW & - & - & 6.21 & 7.03 & - & - & 7.19 & 7.09 & 6.31 & 7.00 \\
\hline $\mathrm{Fe} I$ & 649.6465 & -0.570 & FMW & 7.25 & 6.836 & 6.99 & 6.73 & 7.39 & 6.97 & - & - & 7.49 & 6.76 \\
\hline $\mathrm{Fe} I$ & 651.8366 & -2.750 & FMW & - & - & 11.40 & 7.02 & - & - & 13.03 & 7.10 & 10.42 & 6.77 \\
\hline $\mathrm{Fe} I$ & 659.7559 & -1.070 & FMW & - & - & 5.86 & 7.02 & - & - & 6.54 & 7.10 & 5.99 & 7.00 \\
\hline $\mathrm{Fe}_{\mathrm{I}}$ & 670.3565 & -3.160 & FMW & 7.81 & 6.83 & 9.42 & 6.95 & 8.20 & 7.07 & - & - & - & - \\
\hline $\mathrm{Fe} I$ & 672.5356 & -2.300 & FMW & - & - & 4.39 & 7.06 & - & - & 5.29 & 7.11 & - & - \\
\hline $\mathrm{Fe}_{\mathrm{I}}$ & 673.9521 & -4.950 & FMW & - & - & 8.63 & 6.90 & - & - & 10.55 & 6.91 & - & - \\
\hline $\mathrm{Fe} I$ & 674.6954 & -4.350 & FMW & - & - & 3.42 & 6.92 & - & - & 4.84 & 6.94 & 3.52 & 6.92 \\
\hline $\mathrm{Fe} I$ & 679.3258 & -2.470 & FMW & - & - & 3.78 & 7.08 & - & - & 3.97 & 7.01 & 2.77 & 6.86 \\
\hline $\mathrm{Fe}_{\mathrm{I}}$ & 684.2685 & -1.320 & FMW & - & - & 6.36 & 7.14 & - & - & 6.71 & 7.14 & 6.12 & 7.05 \\
\hline $\mathrm{Fe} I$ & 684.3655 & -0.930 & FMW & - & - & 8.33 & 6.98 & - & - & 8.74 & 6.99 & 8.40 & 6.93 \\
\hline $\mathrm{Fe}_{\mathrm{I}}$ & 685.7250 & -2.150 & FMW & - & - & - & - & - & - & - & - & 4.10 & 6.79 \\
\hline Fe I & 686.2492 & -1.570 & FMW & - & - & 5.15 & 7.06 & - & - & 5.92 & 7.13 & 4.90 & 6.98 \\
\hline $\mathrm{Fe} I$ & 691.6680 & -1.450 & FMW & - & - & 8.68 & 7.02 & - & - & 10.25 & 7.20 & 8.96 & 7.00 \\
\hline $\mathrm{Fe} I$ & 697.6922 & -1.850 & FMW & - & - & 2.44 & 6.85 & - & - & 3.71 & 7.04 & 2.72 & 6.89 \\
\hline $\mathrm{Fe} I$ & 698.8524 & -3.660 & FMW & - & - & - & - & - & - & 12.04 & 7.12 & 10.40 & 7.02 \\
\hline $\mathrm{Fe} I$ & 702.2952 & -1.250 & FMW & - & - & 9.64 & 7.04 & - & - & 10.62 & 7.11 & 8.96 & 6.84 \\
\hline $\mathrm{Fe} I$ & 706.9531 & -4.340 & FMW & - & - & 4.00 & 6.93 & - & - & 5.03 & 6.87 & - & - \\
\hline $\mathrm{Fe} I$ & 721.9682 & -1.690 & FMW & - & - & 8.16 & 7.05 & - & - & 9.27 & 7.14 & 8.74 & 7.08 \\
\hline $\mathrm{Fe}_{\mathrm{I}}$ & 747.6375 & -1.680 & FMW & - & - & 2.71 & 7.00 & - & - & - & - & 3.73 & 7.17 \\
\hline $\mathrm{Fe} I$ & 749.8530 & -2.250 & FMW & - & - & 3.87 & 6.94 & - & - & 5.50 & 7.12 & 4.23 & 6.97 \\
\hline $\mathrm{Fe} I$ & 758.3787 & -1.990 & FMW & - & - & 14.44 & 6.90 & - & - & 15.74 & 6.90 & 15.71 & 7.00 \\
\hline $\mathrm{Fe} I$ & 794.1087 & -2.580 & FMW & - & - & 9.04 & 6.91 & - & - & 10.19 & 6.92 & 9.24 & 6.87 \\
\hline $\mathrm{Fe} I$ & 883.8427 & -1.980 & FMW & - & - & 17.12 & 6.92 & - & - & - & - & 18.18 & 6.97 \\
\hline Fe II & 483.3197 & -4.780 & FMW & 2.22 & 6.84 & - & - & - & - & - & - & - & - \\
\hline $\mathrm{Fe}$ II & 499.3358 & -3.650 & FMW & - & - & 6.27 & 7.121 & 4.65 & 6.88 & - & - & - & - \\
\hline $\mathrm{Fe}$ II & 510.0664 & -4.370 & FMW & 3.54 & 6.96 & - & - & - & - & - & - & - & - \\
\hline Fe II & 513.2669 & -4.180 & FMW & - & - & 3.50 & 7.013 & 3.01 & 7.01 & - & - & - & - \\
\hline Fe II & 525.6937 & -4.250 & K88 & 3.08 & 6.82 & 2.90 & 7.035 & - & - & - & - & - & - \\
\hline Fe II & 526.4812 & -3.190 & FMW & 5.71 & 6.79 & 4.55 & 6.809 & 4.56 & 6.90 & - & - & - & - \\
\hline $\mathrm{Fe}$ II & 599.1376 & -3.557 & K88 & - & - & 3.23 & 6.798 & - & - & 3.90 & 7.04 & 3.91 & 6.849 \\
\hline Fe II & 608.4111 & -3.808 & K88 & - & - & 2.74 & 6.977 & - & - & 2.23 & 6.87 & - & - \\
\hline Fe II & 614.9258 & -2.724 & K88 & - & - & 3.16 & 6.864 & 2.75 & 6.79 & 3.58 & 7.10 & 4.17 & 7.000 \\
\hline Fe II & 624.7557 & -2.329 & K88 & - & - & 4.50 & 6.828 & - & - & 4.32 & 6.92 & 5.80 & 6.979 \\
\hline Fe II & 636.9462 & -4.253 & K88 & - & - & 2.28 & 6.918 & - & - & 2.43 & 7.01 & 2.84 & 6.970 \\
\hline $\mathrm{Fe}$ II & 643.2680 & -3.500 & H92 & - & - & 4.60 & 6.760 & - & - & 4.50 & 7.02 & - & 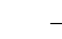 \\
\hline Fe II & 645.6383 & -2.075 & K88 & - & - & 5.87 & 6.907 & - & - & 4.90 & 6.84 & - & - \\
\hline Fe II & 651.6080 & -3.380 & H92 & - & - & 6.08 & 6.953 & - & - & 5.65 & 7.02 & 6.81 & 7.021 \\
\hline
\end{tabular}


Table 5. continued.

\begin{tabular}{|c|c|c|c|c|c|c|c|c|c|c|c|c|c|}
\hline Ion & $\begin{array}{r}\lambda \\
(\mathrm{nm})\end{array}$ & $\log g f$ & $\begin{array}{l}\text { Source of } \\
\log g f \\
\text { (see notes) }\end{array}$ & $\begin{array}{r}E W \\
(\mathrm{pm})^{a} \\
1272\end{array}$ & $\bar{\epsilon}$ & $\begin{array}{r}E W \\
(\mathrm{pm})^{a} \\
1282\end{array}$ & $\epsilon$ & $\begin{array}{r}E W \\
(\mathrm{pm})^{a} \\
1515\end{array}$ & $\bar{\epsilon}$ & $\begin{array}{r}E W \\
(\mathrm{pm})^{a} \\
1665\end{array}$ & $\epsilon$ & $\begin{array}{r}E W \\
(\mathrm{pm})^{a} \\
1708\end{array}$ & $\epsilon$ \\
\hline$\overline{M g} \mathrm{I}$ & 552.8405 & -0.522 & GC & 20.79 & 6.88 & 22.72 & 6.86 & - & - & - & - & - & - \\
\hline $\mathrm{Mg}_{\mathrm{I}}$ & 571.1087 & -1.729 & GC & 12.14 & 7.03 & 12.75 & 6.96 & 12.37 & 7.12 & - & - & - & - \\
\hline $\mathrm{Mg}_{\mathrm{I}}$ & 631.8716 & -1.945 & GC & 2.89 & 6.68 & - & - & 3.81 & 6.91 & - & - & 4.49 & 6.88 \\
\hline Mg I & 631.9237 & -2.165 & GC & 3.04 & 6.93 & 3.30 & 6.91 & 2.79 & 6.93 & 3.54 & 6.88 & 3.31 & 6.90 \\
\hline Mg I & 738.7689 & -1.020 & KP & - & - & - & - & - & - & 4.81 & 6.82 & - & - \\
\hline Mg I & 871.7825 & -0.772 & GC & - & - & 5.40 & 6.85 & - & - & 6.41 & 7.10 & 4.76 & 6.73 \\
\hline$M g_{I}$ & 892.3568 & -1.659 & GC & - & - & 4.28 & 6.84 & - & - & - & - & - & - \\
\hline Si I & 594.8541 & -1.230 & GARZ & - & - & 7.32 & 7.04 & 8.15 & 7.23 & - & - & - & - \\
\hline Si I & 612.5021 & -1.521 & ED & 3.49 & 7.16 & 3.05 & 7.19 & 2.76 & 7.15 & - & - & - & - \\
\hline Si I & 613.1852 & -1.140 & KP & - & - & - & - & - & - & 3.68 & 7.02 & - & - \\
\hline Si I & 614.2482 & -1.480 & ED & 2.37 & 6.87 & 1.97 & 6.88 & 2.70 & 7.10 & 1.97 & 6.92 & - & - \\
\hline Si I & 614.5015 & -1.430 & ED & 2.05 & 6.73 & - & - & 2.85 & 7.08 & 2.17 & 6.93 & - & - \\
\hline Si I & 615.5134 & -0.770 & ED & 5.99 & 6.89 & 6.30 & 7.09 & 6.43 & 7.12 & 5.60 & 7.06 & - & - \\
\hline Si I & 703.4902 & -0.880 & GARZ & - & - & 4.22 & 7.14 & - & - & - & - & 5.05 & 7.22 \\
\hline Si I & 725.0627 & -1.042 & SG & - & - & - & - & - & - & 3.45 & 6.90 & 3.84 & 6.83 \\
\hline Si I & 727.5292 & -1.003 & SG & - & - & 4.07 & 6.90 & - & - & 4.79 & 7.14 & 3.99 & 6.82 \\
\hline Si I & 728.9176 & -0.197 & SG & - & - & - & - & - & - & 7.36 & 6.81 & 7.96 & 6.71 \\
\hline Si I & 793.2349 & -0.470 & GARZ & - & - & 5.33 & 7.05 & - & - & - & - & 5.67 & 7.03 \\
\hline Si I & 874.2446 & -0.630 & $\mathrm{KP}$ & - & - & 6.17 & 7.20 & - & - & 5.51 & 7.21 & 6.88 & 7.23 \\
\hline $\mathrm{Ca} \mathrm{I}$ & 551.2980 & -0.447 & SR & 11.00 & 5.90 & 11.72 & 5.72 & 9.85 & 5.69 & - & - & - & - \\
\hline $\mathrm{Ca} \mathrm{I}$ & 585.7451 & 0.240 & SR & 14.37 & 5.76 & - & - & 14.75 & 5.83 & - & - & - & - \\
\hline $\mathrm{Ca} \mathrm{I}$ & 586.7562 & -1.490 & GC & 4.87 & 5.79 & 4.73 & 5.54 & 4.71 & 5.78 & - & - & - & - \\
\hline $\mathrm{Ca}$ & 616.1296 & -1.266 & SR & 10.22 & 5.98 & - & - & - & - & - & - & - & - \\
\hline $\mathrm{Ca} \mathrm{I}$ & 616.6438 & -1.142 & SR & - & - & 12.19 & 5.87 & 10.97 & 6.05 & 13.90 & 5.90 & - & - \\
\hline $\mathrm{Ca} \mathrm{I}$ & 616.9042 & -0.797 & SR & 12.91 & 6.05 & 13.46 & 5.76 & 11.89 & 5.88 & 15.87 & 5.93 & 13.23 & 5.68 \\
\hline $\mathrm{Ca} \mathrm{I}$ & 643.9075 & 0.390 & SR & 18.79 & 5.80 & 21.41 & 5.73 & 18.69 & 5.76 & - & - & 20.87 & 5.64 \\
\hline $\mathrm{Ca} \mathrm{I}$ & 645.5597 & -1.290 & SR & - & - & 10.88 & 5.75 & 9.57 & 5.90 & 11.94 & 5.63 & 10.31 & 5.63 \\
\hline $\mathrm{Ca} \mathrm{I}$ & 649.3781 & -0.109 & SR & 16.15 & 5.87 & 17.72 & 5.72 & 15.70 & 5.84 & 19.39 & 5.71 & 17.09 & 5.57 \\
\hline $\mathrm{Ca} \mathrm{I}$ & 649.9650 & -0.818 & SR & 12.17 & 5.87 & 13.96 & 5.81 & 12.22 & 5.94 & 15.41 & 5.77 & 13.59 & 5.70 \\
\hline Ti I & 488.5078 & 0.358 & MFW & - & - & - & - & 10.72 & 4.46 & - & - & - & - \\
\hline Ti I & 491.5229 & -1.019 & MFW & 5.14 & 4.58 & - & - & 4.06 & 4.44 & 8.68 & 4.45 & 5.49 & 4.30 \\
\hline Ti I & 499.7096 & -2.118 & MFW & 11.51 & 4.35 & 14.81 & 4.58 & 11.41 & 4.48 & - & - & - & - \\
\hline Ti I & 508.7058 & -0.780 & MFW & 9.36 & 4.53 & - & - & 9.19 & 4.57 & - & - & - & - \\
\hline Ti I & 586.6450 & -0.840 & MFW & 11.85 & 4.37 & 15.65 & 4.57 & 12.41 & 4.61 & - & - & - & - \\
\hline Ti I & 606.4626 & -1.944 & MFW & - & - & 9.61 & 4.50 & - & - & 13.38 & 4.70 & 9.79 & 4.53 \\
\hline Ti I & 609.2792 & -1.379 & MFW & - & - & 4.73 & 4.40 & - & - & 6.88 & 4.31 & 3.81 & 4.28 \\
\hline Ti I & 612.6215 & -1.425 & MFW & 9.80 & 4.52 & 13.75 & 4.71 & 9.32 & 4.56 & - & - & - & - \\
\hline Ti I & 631.2236 & -1.552 & MFW & - & - & 7.91 & 4.41 & - & - & 10.52 & 4.36 & 6.76 & 4.27 \\
\hline Ti I & 633.6098 & -1.743 & MFW & - & - & 7.34 & 4.50 & - & - & 10.38 & 4.52 & 6.98 & 4.47 \\
\hline Ti I & 655.6061 & -1.074 & MFW & - & - & 12.51 & 4.62 & - & - & 16.40 & 4.86 & 10.88 & 4.35 \\
\hline Ti I & 659.9105 & -2.085 & MFW & - & - & - & - & - & - & 15.41 & 4.83 & 9.88 & 4.38 \\
\hline Ti I & 686.1447 & -0.740 & MFW & - & - & 4.95 & 4.27 & - & - & 7.42 & 4.25 & 5.03 & 4.31 \\
\hline Ti I & 718.8565 & -1.760 & MFW & - & - & 7.04 & 4.37 & - & - & - & - & 5.73 & 4.22 \\
\hline Ti I & 744.0578 & -0.700 & MFW & - & - & 5.15 & 4.20 & - & - & - & - & 4.88 & 4.19 \\
\hline Ti I & 802.4843 & -1.140 & MFW & - & - & - & - & - & - & - & - & 7.61 & 4.42 \\
\hline Ti I & 835.3161 & -2.677 & MFW & - & - & 9.82 & 4.61 & - & - & 14.72 & 4.75 & 7.99 & 4.42 \\
\hline Ti I & 867.5372 & -1.669 & MFW & - & - & 14.80 & 4.58 & - & - & - & - & 14.29 & 4.49 \\
\hline Ti I & 868.2980 & -1.941 & MFW & - & - & 11.91 & 4.46 & - & - & 17.32 & 4.72 & 11.89 & 4.46 \\
\hline Ti I & 869.2331 & -2.295 & MFW & - & - & 10.48 & 4.63 & - & - & - & - & 10.25 & 4.61 \\
\hline Ti I & 873.4712 & -2.384 & MFW & - & - & 9.15 & 4.56 & - & - & 14.40 & 4.74 & 8.18 & 4.47 \\
\hline Ni I & 493.5830 & -0.350 & FMW & - & - & 7.40 & 5.49 & - & - & - & - & 7.33 & 5.40 \\
\hline $\mathrm{Ni}$ I & 585.7746 & -0.636 & K88 & 4.37 & 5.43 & 5.36 & 5.61 & 4.47 & 5.60 & - & - & - & - \\
\hline $\mathrm{Ni}$ I & 600.7306 & -3.330 & FMW & - & - & 8.42 & 5.48 & - & - & - & - & 9.12 & 5.54 \\
\hline Ni I & 608.6276 & -0.530 & FMW & - & - & 4.01 & 5.37 & - & - & 4.67 & 5.45 & 4.83 & 5.48 \\
\hline $\mathrm{Ni}$ I & 611.1065 & -0.870 & FMW & - & - & - & - & - & - & - & - & 3.47 & 5.33 \\
\hline $\mathrm{Ni}$ I & 612.8963 & -3.330 & FMW & - & - & 9.04 & 5.58 & 6.28 & 5.44 & - & - & - & - \\
\hline Ni I & 613.0130 & -0.960 & FMW & 1.43 & 5.16 & - & - & 2.25 & 5.55 & - & - & - & - \\
\hline Ni I & 617.5360 & -0.530 & FMW & 5.01 & 5.33 & 5.32 & 5.39 & 5.35 & 5.55 & 5.34 & 5.33 & 5.93 & 5.44 \\
\hline Ni I & 617.6807 & -0.260 & WL & 6.42 & 5.33 & 7.04 & 5.44 & 6.77 & 5.56 & - & - & - & - \\
\hline Ni I & 617.7236 & -3.500 & FMW & 4.99 & 5.37 & 5.66 & 5.36 & 4.96 & 5.57 & 7.39 & 5.41 & - & - \\
\hline $\mathrm{Ni}$ I & 618.6708 & -0.960 & FMW & - & - & - & - & - & - & - & - & 3.30 & 5.41 \\
\hline Ni I & 620.4600 & -1.100 & WL & - & - & 2.46 & 5.38 & - & - & 2.84 & 5.39 & 2.83 & 5.43 \\
\hline $\mathrm{Ni}$ I & 632.7592 & -3.150 & FMW & - & - & - & - & - & - & - & - & 11.26 & 5.69 \\
\hline $\mathrm{Ni}$ I & 648.2796 & -2.630 & FMW & - & - & 10.77 & 5.51 & - & - & 12.10 & 5.51 & 10.99 & 5.46 \\
\hline $\mathrm{Ni}$ I & 658.6307 & -2.810 & FMW & - & - & - & - & - & - & 12.39 & 5.76 & 11.33 & 5.72 \\
\hline Ni I & 659.8592 & -0.980 & FMW & - & - & - & - & - & - & - & - & 2.88 & 5.50 \\
\hline $\mathrm{Ni}$ I & 677.2313 & -0.980 & FMW & - & - & 6.62 & 5.47 & - & - & 7.12 & 5.47 & 7.59 & 5.57 \\
\hline Ni I & 700.1534 & -3.660 & FMW & - & - & - & - & - & - & - & - & 5.49 & 5.55 \\
\hline Ni I & 706.2951 & -3.500 & FMW & - & - & 5.77 & 5.50 & - & - & 7.98 & 5.61 & 6.18 & 5.52 \\
\hline Ni I & 778.8936 & -2.420 & FMW & - & $\begin{array}{lll}- \\
-\end{array}$ & - & - & - & $\begin{array}{lll}- \\
-\end{array}$ & - & - & 14.92 & 5.71 \\
\hline
\end{tabular}

a $1 \mathrm{pm}=10^{-12} \overline{\mathrm{m}}$.

ED Edvardsson et al. (1993); FMW Fuhr et al. (1988); GARZ Garz (1973); GC Gratton et al. (2003); H92 Hannaford et al. (1992); K88 Kurucz (1988); KP Kurucz et al. (1975); MFW Martin et al. (1988); NBS Wiese et al. (1966); SG Schulz-Gulde (1969); WL Wickliffe \& Lawler (1997). 